\title{
Características definidoras dos diagnósticos de enfermagem delineados para recém-nascidos hospitalizados: uma revisão integrativa
}

\author{
Defining characteristics of nursing diagnostics designed for hospitalized newborn: an \\ integrative review
}

\section{Definición de las características del diagnóstico de enfermería diseñado para el recién nacido hospitalizado: una revisión integrativa}

Bruna Ferreira Mendonça Silva ${ }^{1}$, Suely Amorim de Araújo', Tatiany Calegari ${ }^{1 *}$.

\begin{abstract}
RESUMO
Objetivo: Identificar as características definidoras dos diagnósticos de enfermagem delineados para os recémnascidos hospitalizados. Métodos: Revisão integrativa da literatura realizada nas bases de dados Biblioteca Virtual em Saúde (BVS), Literatura Latino-Americana e do Caribe em Ciências da Saúde (LILACS) e Cumulative Index to Nursing and Allied Health Literature (CINAHL) com os Descritores em Ciências da Saúde (DeCS): "Diagnóstico de Enfermagem" AND "Recém-Nascido"; na base Medical Literature Analysis and Retrieval System Online (MEDLINE) foram usados os termos controlados Medical Subject Heading (MeSH): "Nursing Diagnosis" AND "Infant, Newborn". Critérios de inclusão: idiomas inglês e português, artigos primários, publicados entre 2010-2020, disponíveis na íntegra, que atendessem ao objetivo proposto. Resultados: Nos sete estudos selecionados, os diagnósticos de enfermagem foram referentes aos temas: amamentação, hipertermia, icterícia, problemas respiratórios, hipotermia, glicemia e cardiopatias, agrupados nas categorias temáticas "Acurácia das características definidoras para o desenvolvimento de diagnósticos de enfermagem do recém-nascido" e "Características definidoras conceituais refinadas para a prática clínica no cuidado ao recém-nascido". Considerações finais: $O$ diagnóstico de enfermagem adequado está relacionado à expertise do enfermeiro em examinar o recém-nascido, identificar sinais e sintomas, delinear as características definidoras, direcionando para intervenções apropriadas e qualidade da assistência ao recém-nascido hospitalizado.
\end{abstract}

Palavras-chave: Diagnóstico de enfermagem, Recém-nascido, Cuidados de enfermagem.

\begin{abstract}
Objective: Identify studies on the defining characteristics of Nursing Diagnoses outlined for hospitalized newborns. Methods: Integrative Literature Review conducted in the Biblioteca Virtual em Saúde (BVS), Literatura Latino-Americana e do Caribe em Ciências da Saúde (LILACS) and Cumulative Index to Nursing and Allied Health Literature (CINAHL) databases with Science Descriptors of Health: "Diagnóstico de Enfermagem" AND "RecémNascido"; in the Medical Literature Analysis and Retrieval System Online (MEDLINE) database, the controlled terms Medical Subject Heading (MeSH) were used: "Nursing Diagnosis" AND "Infant, Newborn". Inclusion criteria: English and Portuguese languages, primary articles, published between 2010-2020, available in full, that met the proposed objective. Results: In the seven selected studies, nursing diagnoses were related to the themes: breastfeeding, hyperthermia, jaundice, respiratory problems, hypothermia, blood glucose and heart diseases, grouped in the thematic categories "Accuracy of defining characteristics for the development of nursing diagnoses for the newborn" and "Characteristics refined conceptual definers for clinical practice in newborn care". Final Considerations: The proper nursing diagnosis is related to the nurse's expertise in examining the newborn, identifying signs and symptoms, outlining the defining characteristics, directing to appropriate interventions and quality of care for hospitalized newborns.
\end{abstract}

Keywords: Nursing diagnosis, Newborn, Nursing care.

\section{RESUMEN}

Objetivo: Identificar estudios sobre las características definitorias de los diagnósticos de enfermería descritos para los recién nacidos hospitalizados. Métodos: Revisión integral de la literatura realizada en la Biblioteca Virtual de Salud (BVS), Literatura Latinoamericana y del Caribe en Ciencias de la Salud (LILACS) y bases de datos del

${ }^{1}$ Universidade Federal de Uberlândia (UFU), Uberlândia - MG. `E-mail: calegari.tatiany@gmail.com 
Índice Acumulativo de Enfermería y Literatura Aliada en Salud (CINAHL) con Descriptores de Ciencias de la Salud: "Diagnóstico de Enfermería" Y "Bebé, recién nacido"; en la base de datos del Medical Literature Analysis and Retrieval System Online (MEDLINE), se utilizaron los términos controlados Medical Subject Heading (MeSH): "Nursing Diagnosis" Y "Infant, Newborn". Criterios de inclusión: inglés y portugués, artículos primarios, publicados entre 2010 y 2020, disponibles en su totalidad, que cumplieron con el objetivo propuesto. Resultados: En los siete estudios seleccionadoslos diagnósticos de enfermería se relacionaron con los temas: lactancia materna, hipertermia, ictericia, problemas respiratorios, hipotermia, glucosa en sangre y enfermedades del corazón, agrupados en las categorías temáticas "Precisión de definir características para el desarrollo de diagnósticos de enfermería para el recién nacido" y "Características definitorias conceptuales refinadas para la práctica clínica en la atención neonatal”. Consideraciones finales: El diagnóstico de enfermería adecuado está relacionado con la experiencia de la enfermera en el examen del recién nacido, identificando signos y síntomas, describiendo las características definitorias, dirigiendo las intervenciones apropiadas y la calidad de la atención para los recién nacidos hospitalizados.

Palabras clave: Diagnóstico de enfermería, Recién nacido, Atención de enfermería.

\section{INTRODUÇÃO}

A Sistematização da Assistência de Enfermagem (SAE) é uma metodização utilizada para que a assistência de enfermagem seja prestada de forma eficaz e vise operacionalizar esta premissa e foi necessária para que os profissionais de enfermagem criassem meios facilitadores do processo de organização do cuidado de forma individualizada. A SAE caracteriza-se como um sistema organizado o qual padroniza o cuidado, a partir de pressupostos científicos. É um instrumento privativo de responsabilidade do enfermeiro, que tem como objetivos a identificação do problema, o reconhecimento de intervenções de prevenção e promoção da saúde do ser humano, ao desenvolver ações individualizadas e permitir resultados alcançáveis (TRUPPEL TC, et al., 2009).

A Resolução do Conselho Federal de Enfermagem número 358 (2009) dispõe sobre a SAE e a implementação do Processo de Enfermagem em ambientes, públicos ou privados, nos quais ocorre o cuidado profissional de Enfermagem. A SAE se organiza em cinco etapas inter-relacionadas, interdependentes e recorrentes, que são: a coleta de dados de enfermagem ou histórico de enfermagem, diagnóstico de enfermagem, planejamento de enfermagem, implementação das ações e intervenções, avaliação de enfermagem (COFEN, 2009).

O diagnóstico de enfermagem (DE) é a segunda etapa do Processo de Enfermagem, considerado pelos profissionais de enfermagem a real representação do problema. É um método de raciocínio, de avaliação e julgamento, que exige um processo cognitivo e habilidades intelectuais, além de vivência prática e conhecimento científico. O DE é baseado na presença de indícios e/ou inferências clínicas, passíveis de serem observadas, e na análise de fatores relacionados ao fenômeno em observação, que podem ocorrer tanto com o cliente, com a família ou com a comunidade (FREITAS LJQ, et al., 2014).

A determinação da sensibilidade, especificidade e poder preditivo dos sinais clínicos associados com um DE é uma parte importante de validação de diagnósticos de enfermagem, que por sua vez é uma etapa essencial do Processo de Enfermagem, o qual ainda é composto pela avaliação, o diagnóstico, a intervenção e a avaliação de resultados.

Os DE precisos contribuem para elevar a qualidade dos cuidados de saúde e redução dos custos em instituições de saúde. Para se estabelecer de forma rigorosa o DE é preciso identificar indicadores clínicos preditivos desses diagnósticos (PARKER L e LUNNEY M, 1998). Portanto, para aumentar a probabilidade de pacientes com diagnóstico específico estarem devidamente identificados, é necessário desenvolver um conjunto de indicadores observáveis que se agrupam como manifestações de um diagnóstico, que são as chamadas características definidoras (CD).

Para se chegar a um DE o exame físico é fundamental, pois é a partir desta ação que se faz uma coleta de dados ampla, a qual pode contribuir para a sua acurácia. A identificação e a validação das características definidoras que são utilizadas para estabelecer o diagnóstico correto se tornam cruciais para compreender melhor o processo diagnóstico (SILVEIRA UA, et al., 2008). 
Dentre os sistemas de classificação de DE, destaca-se a taxonomia da North American Nursing Diagnosis Association (NANDA), que é um dos mais aplicados e utilizados mundialmente no processo do raciocínio e do julgamento clínico acerca dos problemas de saúde ou dos processos vitais. Não obstante, o reconhecimento do valor do diagnóstico de enfermagem como instrumento para raciocínio e julgamento, ainda há uma dimensão de experiência ou resposta que é objeto desse processo cognitivo, o conceito diagnóstico em si (HERDMAN TH e KAMITSURU S, 2018).

Profissionais treinados e capacitados são capazes de identificar os indicadores observáveis, mais conhecidos como as características definidoras de forma rápida e precisa, direcionando para o correto diagnóstico dos clientes no âmbito hospitalar, inclusive o recém-nascido $(R N)$, direcionando para o início do tratamento adequado de cada diagnóstico traçado, propiciando o restabelecimento e promoção de sua saúde.

Diante das informações disponíveis na perspectiva das características definidoras dos DE para RN surgiu a pergunta norteadora: quais as evidências científicas em relação aos $\mathrm{RN}$ hospitalizados, que utilizam das características definidoras para estabelecer diagnósticos de enfermagem, visando a melhor assistência de enfermagem ao RN. O presente estudo tem como objetivo identificar as características definidoras dos diagnósticos de enfermagem delineados para os $\mathrm{RN}$ hospitalizados.

\section{MÉTODOS}

Trata-se de uma pesquisa de Revisão Integrativa da literatura. Para elaborar uma Revisão Integrativa significativa que poderá contribuir com a implementação de intervenções eficazes no cuidado aos pacientes, é necessário que as etapas a serem seguidas estejam claramente descritas. Nesta construção é preciso percorrer seis etapas diferentes, semelhantes aos estágios de desenvolvimento de pesquisa convencional. As etapas são: identificação do tema e seleção da hipótese ou questão de pesquisa para a elaboração da Revisão Integrativa; estabelecimento de critérios para inclusão e exclusão de estudos/ amostragem ou busca na literatura; definição das informações a serem extraídas dos estudos selecionados/categorização; avaliação dos estudos incluídos; interpretação dos resultados; apresentação da revisão/síntese do conhecimento (MENDES KDS, et al., 2008).

Foi utilizada a estratégia PICO que retrata um acrônimo para Paciente: RN hospitalizados; Intervenção: diagnósticos de enfermagem; Comparação: características definidoras e Outcomes/Desfecho: promoção da melhor assistência de enfermagem ao RN.

A técnica PICO pode ser utilizada para levantar questões de pesquisa de diversas propriedades, provenientes da clínica, do gerenciamento de recursos humanos e materiais, da busca de ferramentas para avaliação de sintomas, entre outras. A pergunta de pesquisa bem construída possibilita a definição correta de que evidências são necessárias para a resolução da questão clínica, potencializa a recuperação de evidências nas bases de dados, enfatiza a finalidade da pesquisa e evita a realização de buscas irrelevantes (SANTOS CMC, et al., 2007).

A busca foi realizada de março a maio de 2020, mediante acesso à BVS, MEDLINE, LILACS e CINAHL. Foram utilizados os DeCS: "Diagnóstico de Enfermagem", "Recém-Nascido", intercalados com operador booleano AND. Na base MEDLINE foram usados os termos controlados MeSH: "Nursing Diagnosis" AND "Infant, Newborn".

Os critérios de inclusão determinantes para a seleção dos artigos foram: artigos primários, publicados entre 2010-2020, disponíveis na íntegra, que atendessem ao objetivo do estudo, nos idiomas inglês e português. Os critérios de exclusão foram estudos repetidos, revisões, teses e aqueles que não atendessem aos objetivos propostos.

Para a seleção dos estudos, foram seguidas as recomendações do Preferred Reporting Items for Systematic reviews and Meta-Analyses (PRISMA). O propósito do PRISMA é facilitar para os autores o relato de revisões sistemáticas e meta-análises, sendo útil para a avaliação crítica destas publicações. 
Mesmo a essência referindo-se a ensaios clínicos randomizados, o PRISMA também pode ser aplicado como um apoio para relatos de revisões sistemáticas de outros tipos de pesquisa, em especial avaliações de intervenções. Entretanto, o checklist PRISMA não é um instrumento de apreciação de qualidade para considerar a virtude de uma revisão sistemática (GALVÃO TF, et al., 2015).

Foram localizados 853 artigos e após aplicados os critérios de inclusão, foram excluídos 493 artigos de outro período de publicação estabelecido, 173 repetidos, nove revisões da literatura e 168 que não atenderam aos objetivos propostos. Realizada leitura aprofundada e selecionados sete artigos, indexados nas bases eletrônicas MEDLINE (71,4\%) e BVS (28,6\%) (Figura 1).

Figura 1 - Fluxograma do processo de seleção dos artigos incluídos na Revisão Integrativa.
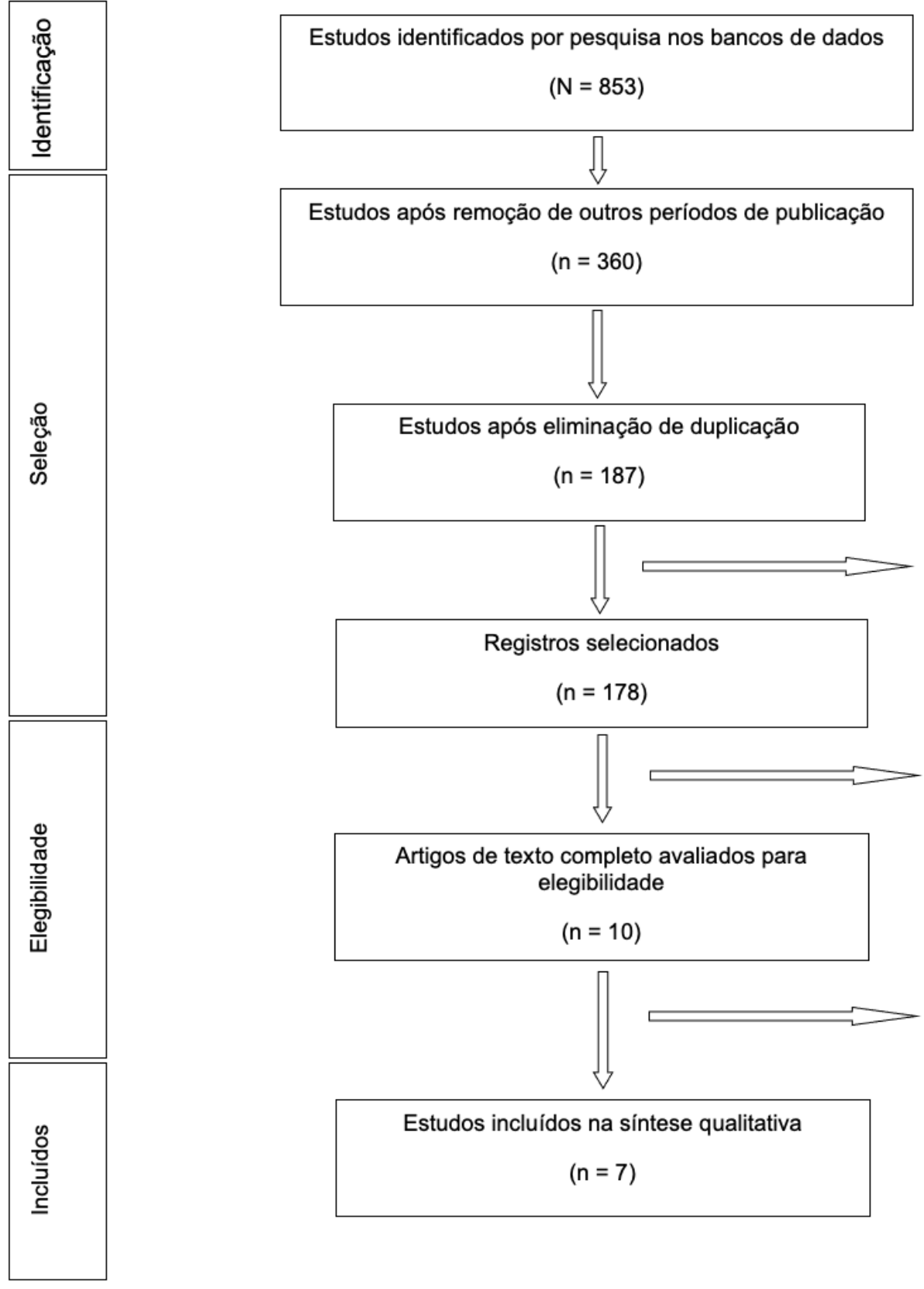

Estudos após remoção de outros períodos de publicação

$(n=360)$
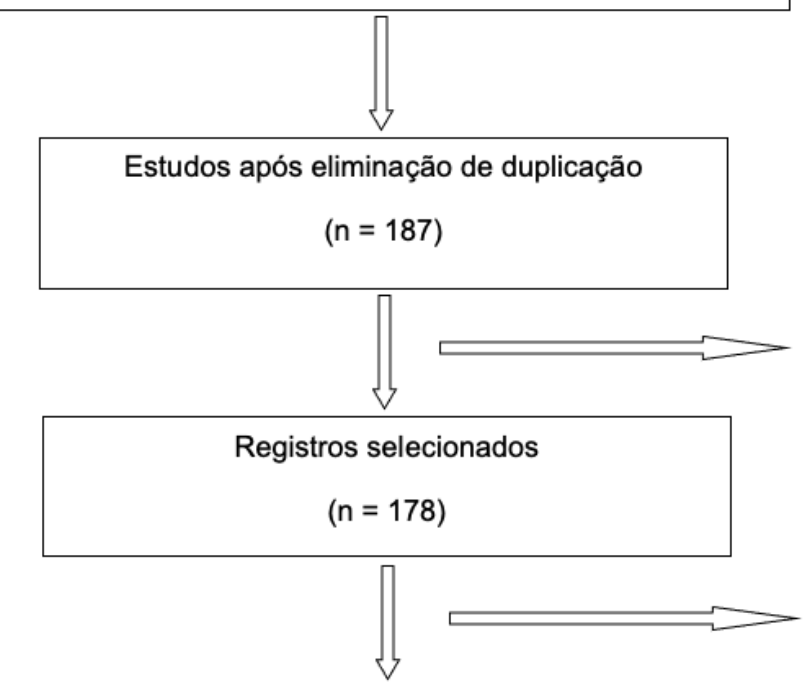

Artigos excluídos:

$(n=9$ revisões $d a$ literatura)

Artigos de texto completo avaliados para elegibilidade

(n=10)

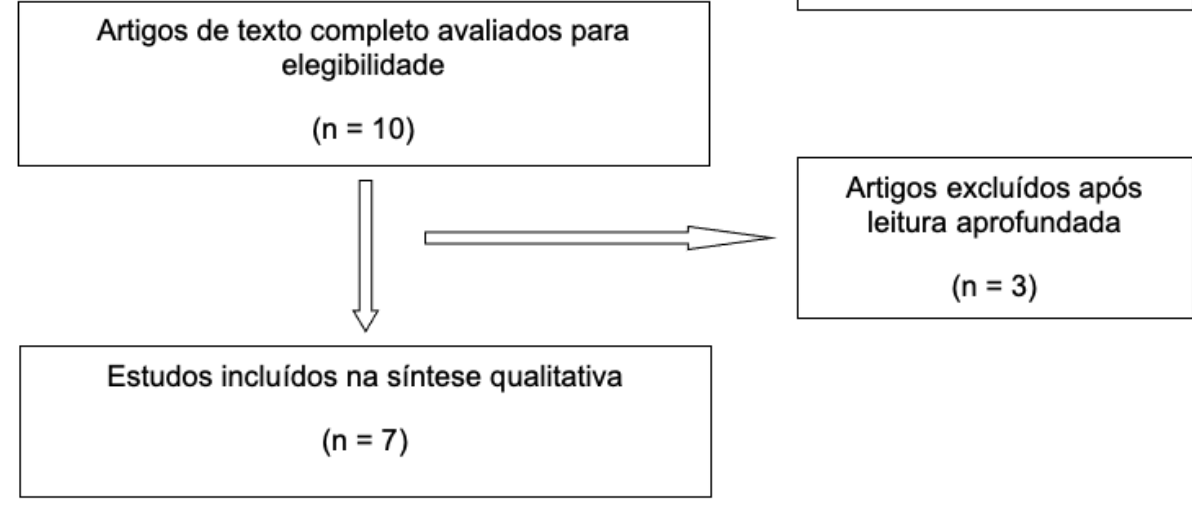

Artigos que não atenderam ao objetivo

( $n=168)$

$(n=3)$

Fonte: Silva BFM, et al., 2020.

Para o desenvolvimento da análise do conteúdo dos estudos, foi elaborado pelas autoras um formulário adaptado de Ursi ES (2005) com os seguintes itens: nome do artigo, nome dos autores, qual a base de dados utilizada, objetivo do estudo, tipo de estudo, resultados encontrados e conclusão. 
Os resultados foram analisados e serão apresentados de forma descritiva. De acordo com o delineamento metodológico proposto não houve necessidade de avaliação do Comitê de Ética em Pesquisa com Seres Humanos.

\section{RESULTADOS E DISCUSSÃO}

Os sete artigos localizados nas bases de dados sobre as características definidoras dos DE foram referentes aos temas: amamentação, hipertermia, icterícia neonatal, problemas respiratórios, hipotermia, glicemia e cardiopatias congênitas. Destes, $71,4 \%$ foram publicados em inglês e $28,6 \%$ em português. Todos os estudos foram realizados em cidades brasileiras.

O estudo de Aquino WKM, et al. (2018) sobre o DE de Hipertermia foi desenvolvido em unidades neonatais de médio e alto risco. Como forma de coleta de dados, foi construído um instrumento, para facilitar e sistematizar o processo.

A investigação de Dantas AVVC, et al. (2018) sobre o DE de Icterícia Neonatal foi realizado com 100 RN com idades entre 24 horas e dez dias de vida, em um hospital público terciário, por meio de um instrumento revisado e reajustado para esta pesquisa.

A publicação de Alvarenga SC, et al. (2018) sobre o DE de Amamentação Ineficaz investigou uma amostra de 73 binômios mãe-filho internados na maternidade de um hospital universitário com utilização de instrumento criado pelos próprios autores.

O estudo de Braga FC, et al. (2014) voltou-se para os achados da acurácia dos indicadores clínicos dos DE Hipertermia e Hipotermia em RN. Estudo realizado em maternidade de referência em Fortaleza, CE, com 46 RN mediante uso de instrumento construído baseado nos indicadores clínicos dos diagnósticos pesquisados.

A pesquisa de Avena MJ, et al. (2014) sobre três DE respiratórios por meio da análise de consenso de um comitê de cinco enfermeiras especialistas em cuidados intensivos em neonatologia e peditria, além de cinco profissionais não enfermeiros com experiência em atenção respiratória aos RN. Após duas rodadas de avaliação, as definições foram validadas.

O estudo de Oliveira SIM, et al. (2013) identificou os DE mais frequentes entre RN com alterações glicêmicas internados em uma maternidade-escola. A publicação de Urakawa IT e Kobayashi RM (2012) foi sobre os DE relacionados à cardiopatia congênita em neonatos de maioria do sexo masculino (61\%), com idade média de 15,5 dias e as patologias mais prevalentes foram comunicação interatrial (48\%), persistência do canal arterial (33\%) e comunicação interventricular (30\%). O detalhamento dos estudos está apresentado a seguir (Quadro 1). 


\section{Revista Eletrônica Acervo Saúde / Electronic Journal Collection Health | ISSN 2178-2091}

Quadro 1 - Dados da revisão integrativa referente às características dos estudos selecionados: nome do artigo, autores, base de dados indexada, objetivo, tipo de estudo, resultados e conclusão.

\begin{tabular}{|c|c|c|c|c|c|c|}
\hline Nome do artigo & Autores & $\begin{array}{c}\text { Base de } \\
\text { dados }\end{array}$ & Objetivo & $\begin{array}{l}\text { Tipo de } \\
\text { estudo }\end{array}$ & Resultados & Conclusão \\
\hline $\begin{array}{l}\text { A1 - Accuracy of } \\
\text { the defining } \\
\text { characteristics in } \\
\text { nursing } \\
\text { diagnoses of } \\
\text { Hyperthermia in } \\
\text { newborns. }\end{array}$ & $\begin{array}{c}\text { AQUINO } \\
\text { WKM, et al. }\end{array}$ & MEDLINE & $\begin{array}{c}\text { Validar as características } \\
\text { Definidoras do diagnóstico de } \\
\text { Hipertermia do RN. }\end{array}$ & $\begin{array}{l}\text { Estudo } \\
\text { transversal }\end{array}$ & $\begin{array}{l}\text { As características sensibilidade, vasodilatação, } \\
\text { Irritabilidade e letargia, são um forte indicativo } \\
\text { da presença de hipertermia }\end{array}$ & $\begin{array}{c}\text { A hipertermia está relacionada } \\
\text { à presença das quatro } \\
\text { características com alta } \\
\text { especificidade, entretanto, a } \\
\text { característica estupor } \\
\text { foi a única com sensibilidade } \\
\text { significativa para identificação } \\
\text { do diagnóstico em estágio } \\
\text { inicial. }\end{array}$ \\
\hline $\begin{array}{l}\text { A2-Nursing } \\
\text { Diagnosis of } \\
\text { Neonatal } \\
\text { Jaundice: Study } \\
\text { of Clinical } \\
\text { Indicators. }\end{array}$ & $\begin{array}{l}\text { DANTAS } \\
\text { AVVC, } \\
\text { et al. }\end{array}$ & MEDLINE & $\begin{array}{l}\text { Identificar as características } \\
\text { definidoras e examinar sua } \\
\text { associação com o diagnóstico } \\
\text { de enfermagem de Icterícia } \\
\text { Neonatal em RN hospitalizado. }\end{array}$ & $\begin{array}{l}\text { Estudo } \\
\text { transversal }\end{array}$ & $\begin{array}{l}\text { As características definidoras mais frequentes } \\
\text { para o DE de Icterícia Neonatal foram cor de } \\
\text { pele amarelo-alaranjada (65\%) e perfil } \\
\text { sanguíneo anormal }(75 \%) \text {. As mucosas } \\
\text { amareladas apresentaram as melhores } \\
\text { medidas de acurácia diagnóstica. }\end{array}$ & $\begin{array}{l}\text { Os indicadores clínicos que } \\
\text { melhor previram e aumentaram } \\
\text { a probabilidade de desenvolver } \\
\text { icterícia foram identificados. }\end{array}$ \\
\hline $\begin{array}{l}\text { A3- Critical } \\
\text { defining } \\
\text { characteristics for } \\
\text { nursing diagnosis } \\
\text { about ineffective } \\
\text { breastfeeding. }\end{array}$ & $\begin{array}{l}\text { ALVAREN } \\
\text { GA SC, et } \\
\quad \text { al. }\end{array}$ & MEDLINE & $\begin{array}{l}\text { Investigar as medidas de } \\
\text { acurácia diagnóstica de } \\
\text { enfermagem e propor um } \\
\text { modelo para uso de } \\
\text { características definidoras no } \\
\text { julgamento do DE de } \\
\text { Amamentação Ineficaz. }\end{array}$ & $\begin{array}{l}\text { Estudo } \\
\text { transversal }\end{array}$ & $\begin{array}{l}\text { Características definidoras para o DE: } \\
\text { descontinuidade da sucção da mama; } \\
\text { incapacidade do lactente de apreender a } \\
\text { região aréolo-mamilar corretamente; } \\
\text { ocorrência de choro do lactente na primeira } \\
\text { hora após a amamentação e suprimento de } \\
\text { leite inadequado percebido. }\end{array}$ & $\begin{array}{l}\text { O processo de amamentação é } \\
\text { dinâmico e o julgamento } \\
\text { diagnóstico pode sofrer } \\
\text { modificações conforme o tempo } \\
\text { em que o dado é coletado. As } \\
\text { características definidoras são } \\
\text { melhores preditoras se } \\
\text { associadas com modelos e } \\
\text { regras de utilização. }\end{array}$ \\
\hline
\end{tabular}

REAS / EJCH | Vol.12(10) | e4328 | DOI: https://doi.org/10.25248/reas.e4328.2020 Página 6 de 10 


\begin{tabular}{|c|c|c|c|c|c|c|}
\hline $\begin{array}{l}\text { A4 - Accuracy of } \\
\text { clinical indicators } \\
\text { of Nursing } \\
\text { Diagnoses } \\
\text { hyperthermia and } \\
\text { hypothermia in } \\
\text { newborns }\end{array}$ & $\begin{array}{l}\text { BRAGA } \\
\text { FC, } \\
\text { et al. }\end{array}$ & MEDLINE & $\begin{array}{c}\text { Identificar os principais } \\
\text { indicadores clínicos do DE } \\
\text { Hipertermia e Hipotermia em } \\
\text { RN. }\end{array}$ & $\begin{array}{l}\text { Estudo } \\
\text { transversal }\end{array}$ & $\begin{array}{l}\text { Para Hipotermia a temperatura abaixo dos } \\
\text { parâmetros normais foi o único indicador } \\
\text { sensível encontrado. Os indicadores clínicos } \\
\text { específicos para Hipotermia foram: } \\
\text { irritabilidade, inquietação, bradicardia, palidez, } \\
\text { icterícia, taquicardia, preenchimento capilar } \\
\text { lento e vasoconstrição periférica. Não foram } \\
\text { identificados indicadores sensíveis para } \\
\text { Hipertermia, sendo a taquipneia, vasodilatação } \\
\text { e apneia os indicadores específicos. }\end{array}$ & $\begin{array}{c}\text { Os achados da presente } \\
\text { pesquisa podem colaborar para } \\
\text { a inferência correta e rápida de } \\
\text { hipotermia e hipertermia em } \\
\text { RN. }\end{array}$ \\
\hline $\begin{array}{l}\text { A5- Conceptual } \\
\text { validation of the } \\
\text { defining } \\
\text { characteristics of } \\
\text { respiratory } \\
\text { nursing } \\
\text { diagnoses in } \\
\text { neonates. }\end{array}$ & $\begin{array}{l}\text { AVENA } \\
\text { MJ, et al. }\end{array}$ & MEDLINE & $\begin{array}{l}\text { Elaborar e validar definições } \\
\text { conceituais e operacionais } \\
\text { para as características } \\
\text { definidoras de três DE } \\
\text { respiratórios para RN: Padrão } \\
\text { Respiratório Ineficaz, Troca de } \\
\text { Gases Prejudicada, Ventilação } \\
\text { Espontânea Prejudicada }\end{array}$ & $\begin{array}{l}\text { Estudo } \\
\text { metodológico }\end{array}$ & $\begin{array}{c}\text { As CD dos DE respiratórios para RN tiveram } \\
\text { em comum para o desconforto respiratório: } \\
\text { agitação, batimento de asa de nariz, dispneia, } \\
\text { uso da musculatura acessória, pressão do gás } \\
\text { carbônico no sangue arterial alterada, } \\
\text { hipoxemia. Gemido expiratório e murmúrio } \\
\text { vesicular dimunuído foram destacados como } \\
\text { relevantes. }\end{array}$ & $\begin{array}{l}\text { As CD dos três DE respiratórios } \\
\text { foram validadas via consenso } \\
\text { de especialistas o que garante } \\
\text { segurança ao enfermeiro na } \\
\text { prática clínica para identificação } \\
\text { dos sinais e sintomas } \\
\text { pertinentes à real situação de } \\
\text { saúde do RN. }\end{array}$ \\
\hline $\begin{array}{l}\text { A6- Diagnósticos } \\
\text { de enfermagem } \\
\text { em recém- } \\
\text { nascido com } \\
\text { alterações } \\
\text { glicêmicas. }\end{array}$ & $\begin{array}{l}\text { OLIVEIRA } \\
\text { SIM, et al. }\end{array}$ & BVS & $\begin{array}{l}\text { Identificar os DE mais } \\
\text { frequentes entre RN com } \\
\text { alterações glicêmicas } \\
\text { internados em uma } \\
\text { maternidade-escola. }\end{array}$ & $\begin{array}{l}\text { Estudo } \\
\text { quantitativo e } \\
\text { documental }\end{array}$ & $\begin{array}{l}\text { Foi observado predomínio de diagnósticos } \\
\text { referentes ao Risco de Crescimento } \\
\text { Desproporcional, Risco de Infecção } \\
\text { e Risco de Glicemia Instável. Estes } \\
\text { relacionaram-se ao peso inadequado em } \\
\text { relação ao percentil para a idade, fatores } \\
\text { nutricionais, gravidade do quadro clínico e } \\
\text { amamentação com dificuldades. }\end{array}$ & $\begin{array}{c}\text { Ressalta-se a importância da } \\
\text { utilização da SAE com o } \\
\text { intuito de planejar o cuidado } \\
\text { aos neonatos com distúrbios } \\
\text { glicêmicos. }\end{array}$ \\
\hline $\begin{array}{l}\text { A7- Identificação } \\
\text { do perfil e } \\
\text { diagnósticos de } \\
\text { enfermagem do } \\
\text { neonato com } \\
\text { cardiopatia } \\
\text { congênita. }\end{array}$ & $\begin{array}{l}\text { URAKAWA } \\
\text { IT e } \\
\text { KOBAYAS } \\
\text { HI RM. }\end{array}$ & BVS & $\begin{array}{l}\text { Caracterizar o perfil e os DE } \\
\text { do neonato com cardiopatia } \\
\text { congênita. }\end{array}$ & $\begin{array}{l}\text { Estudo } \\
\text { descritivo, } \\
\text { exploratório } \\
\text { e } \\
\text { retrospectivo }\end{array}$ & $\begin{array}{c}\text { Os DE prevalentes foram: Risco para } \\
\text { Diminuição do Débito Cardíaco e Instauração } \\
\text { Arterial Periférica. }\end{array}$ & $\begin{array}{c}\text { Conhecer as características do } \\
\text { neonato com doença cardíaca } \\
\text { congênita favorece a prestação } \\
\text { da assistência de enfermagem } \\
\text { direcionadas pelos DE. }\end{array}$ \\
\hline
\end{tabular}

Fonte: SILVA BFM, et al., 2020.

REAS / EJCH | Vol.12(10) | e4328 | DOI: https://doi.org/10.25248/reas.e4328.2020 Página 7 de 10 
Após a leitura aprofundada dos artigos foi possível agrupá-los nas seguintes categorias temáticas: "Acurácia das características definidoras para o desenvolvimento de DE do RN" e "Características definidoras conceituais refinadas para a prática clínica no cuidado ao RN" (Quadro 2).

Quadro 2 - Distribuição dos estudos selecionados de acordo com as categorias temáticas

\begin{tabular}{|l|c|}
\hline \multicolumn{1}{|c|}{ Categoria Tematica } & Artigos \\
\hline Acurácia das características definidoras para o desenvolvimento de DE do RN & A1; A2; A3; A4 \\
\hline $\begin{array}{l}\text { Características definidoras conceituais refinadas para a prática clínica no } \\
\text { cuidado ao RN }\end{array}$ & A5; A6; A7 \\
\hline
\end{tabular}

Fonte: SILVA BFM, et al., 2020.

O padrão normal de temperatura do $\mathrm{RN}$ varia de $36,5^{\circ} \mathrm{C}$ a $37,0^{\circ} \mathrm{C}$ (AQUINO WKM, et al., 2018). A hipertermia neonatal que é definida por temperatura corporal acima dos parâmetros estabelecidos e que ocorre por meio da vasodilatação periférica devido ao empenho do organismo em suprimir o calor merece atenção, pois o cérebro é um órgão bastante sensível às alterações térmicas. Dispositivos com defeito, uso errôneo de lâmpadas de aquecimento, uso de prostaglandinas, septicemia, incubadora próxima ao sol, dentre outros motivos constituem perigo para surgir a hipertermia. As ações do enfermeiro devem ser executadas com base nos sinais clínicos do paciente que permitam a avaliação contínua dos resultados de enfermagem (BRAGA FC, et al., 2014).

O estudo de Aquino VKM, et al. (2018) encontrou as seguintes características definidoras para o DE hipertermia: não manutenção da sucção, pele quente, letargia taquipneia, rubor, hipotonia, taquicardia, irritabilidade, postura anormal, vasodilatação, estupor, apneia e hipotensão. Entretanto, a hipotermia é a temperatura corporal abaixo dos parâmetros normais (menor que $36,5^{\circ} \mathrm{C}$ ). Algumas causas predispõem os $\mathrm{RN}$ à perda de calor, como a grande região de superfície em relação ao peso, isolamento térmico ineficiente devido à fina camada de gordura e o mecanismo de produção de calor, isto é, a termogênese sem tremor. $O$ $\mathrm{RN}$ corresponde ao estresse do esfriamento com a vasoconstrição e a queda na temperatura da pele, que podem ser os primeiros sinais do desenvolvimento da hipotermia (BRAGA FC, et al., 2014).

Devido à dificuldade e à importância da termorregulação para a preservação do $\mathrm{RN}$, é necessário que o enfermeiro possua profundo entendimento do mecanismo de controle térmico e da instabilidade térmica dessa população, uma vez que os cuidados relacionados ao controle e à manutenção da temperatura corporal são fundamentais para sua sobrevida (BRAGA FC, et al., 2014). As características definidoras encontradas para hipotermia foram: temperatura abaixo dos parâmetros normais, cianose nos leitos ungueais, hipóxia, letargia, pele fria, irritabilidade, inquietação, bradicardia, manchas na pele, palidez, piloereção, icterícia, taquicardia, hipotonia, preenchimento capilar lento vasoconstrição periférica (BRAGA FC, et al., 2014).

A icterícia neonatal é a manifestação clínica de hiperbilirrubinemia, cujo acontecimento é resultado de disfunção na predisposição de um RN a produzir e na capacidade de excretar bilirrubina (DANTAS AVVC, et al., 2018). A icterícia neonatal, na maioria das vezes, é definida como um episódio fisiológico passageiro, que começa após as primeiras 24 horas de vida, tendo o seu pico entre o terceiro e o quinto dia de vida nos $\mathrm{RN}$ a termo, perdurando em média por sete dias.

Conforme Machado SPC, et al. (2012), a fototerapia é a terapêutica mais aplicada, no mundo, no tratamento da icterícia neonatal, devido à sua alta efetividade e ausência de efeitos colaterais que expliquem o não uso da mesma. A equipe de enfermagem neste caso cumpre o papel de receber, preparar o RN para a terapêutica e prestar os cuidados pertinentes, bem como, organizar os aparelhos que serão usados para a fototerapia, como os focos de luz, as incubadoras, entre outros (SENA DTC, et al., 2015). As características definidoras mais frequentes para o DE hiperbilirrubinemia neonatal foram: cor de pele amarelo-alaranjado, perfil sanguíneo anormal, membranas mucosas amarelas e escleras amareladas (DANTAS AVVC, et al., 2018).

O aleitamento materno (AM) é reconhecido como o método padrão para a alimentação do bebê, principalmente nos seis primeiros meses de vida, fase na qual é orientada sua prática de forma 
exclusiva. Além das vantagens nutricionais, o AM melhora a resposta imunológica da criança, reduz a incidência de infecções e outras morbidades infantis. Para a mãe, essa prática está associada a um menor risco para o desenvolvimento de sangramento, anemia, câncer de mama e câncer ovariano (NASCIMENTO AMR, 2019).

A amamentação ineficaz ocorre quando existe uma barreira para prover o leite para um lactente ou criança diretamente das mamas, o que pode prejudicar o estado nutricional do lactente ou da criança. A equipe de enfermagem comumente se engaja na orientação e assistência da amamentação, o que exige que sua competência de avaliação e julgamento clínico sejam regularmente aprimoradas (ALVARENGA SC, et al., 2018).Para o DE de Amamentação Ineficaz, foram estabelecidas as seguintes características definidoras: descontinuidade da sucção da mama, incapacidade do lactente de apreender a região aréolomamilar corretamente, ocorrência de choro do lactente na primeira hora após a amamentação e suprimento de leite inadequado percebido (ALVARENGA SC, et al., 2018).

$\mathrm{Na}$ avaliação do quadro clínico dos pacientes, o enfermeiro se depara com o encargo de julgar e eleger o DE que melhor representa um estabelecido conjunto de características definidoras. Contudo, o profissional ainda enfrenta muitos obstáculos em estabelecer a resposta humana mais apropriada, principalmente quando não se conhecem os indicadores clínicos de um fenômeno abrangente e ininterrupto (MONTEIRO FPM, et al., 2016).

A inferência diagnóstica adequada, por meio da apuração de indicadores clínicos sensíveis e específicos para o reconhecimento desses diagnósticos, permite intervenções rápidas e eficazes para obter os resultados esperados. $O$ processo de raciocínio diagnóstico auxilia o enfermeiro na tomada de decisão sobre os DE em uma população. Entretanto, para ocorrer a promoção da assistência, é fundamental a inferência de diagnósticos precisos (BRAGA FC, et al., 2014).

As cardiopatias congênitas são anomalias resultantes de defeitos anatômicos no coração ou na rede circulatória, que comprometem sua função. No período neonatal apenas são diagnosticadas na apresentação precoce por sinais como cianose, insuficiência cardíaca, sopro ou arritmia, sendo usualmente mais graves e causas frequentes de emergência pediátrica. O reconhecimento de defeitos cardíacos é importante devido à sua implicação no prognóstico em virtude da rápida deterioração clínica e da sua alta mortalidade. Os DE nesse contexto favorecem o melhor planejamento da assistência de enfermagem (URAKAWA IT E KOBAYASHI RM, 2012).

Em relação aos aspectos respiratórios dos $\mathrm{RN}$, as dificuldades de adequação à vida extrauterina podem ser apresentadas por muitos prematuros. Os motivos compreendem mais comumente a imaturidade pulmonar e surfactante deficiente, repercutindo em doenças pulmonares crônicas quando necessário 0 cuidado intensivo, a despeito de resultar na melhora da sobrevida destes RN pré-termos. Com esta terapêutica intensiva, consequentemente é acentuada a monitorização e o tratamento específico. Nesta condição, os DE respiratórios, ou seja, aqueles que têm relação direta com a respiração, são de relevância para a população neonatal. O estudo de Avena MJ, et al. (2014) apresenta três detes diagnósticos relacionados ao padrão respiratório e/ou alteração do padrão respiratório normal: Troca de Gases Prejudicada, Padrão Respiratório Ineficaz e Ventilação Espontânea Prejudicada (AVENA MJ, et al., 2014).

O distúrbio glicêmico em neonatos é um tema bastante discutido na literatura em virtude dos agravos que essas alterações podem acarretar para o desenvolvimento dos RN. A hiperglicemia ocorre em $20 \%$ a $85 \%$ dos RN e não há um consenso sobre a sua exata definição. Sugere-se como ponto de corte para hiperglicemia níveis de glicose de $145 \mathrm{mg} / \mathrm{dL}$. A hipoglicemia neonatal acomete de $3 \%$ a $43 \%$ dos neonatos e ocorre principalmente nas primeiras horas de vida. A glicose é ofertada ao feto por gradiente de concentração por meio da placenta. Dessa forma, a glicemia do feto está diretamente relacionada à glicemia materna, ficando em torno de 50 a $55 \mathrm{mg} / \mathrm{dL}$ (OLIVEIRA SIM, et al., 2013).

Esses distúrbios requerem atenção especial da enfermagem por predisporem a danos dependendo de sua gravidade. A partir de estudos, e conhecendo as alterações glicêmicas neonatais, podem-se elaborar DE que norteiem o trabalho da equipe de enfermagem que presta assistência a esses $\mathrm{RN}$, visando reduzir 
as complicações previsíveis advindas desses agravos (OLIVEIRA SIM, et al., 2013). As características definidoras dos $\mathrm{DE}$ direcionadas aos $\mathrm{RN}$ quando bem elucidadas são relevantes ferramentas de auxílio ao enfermeiro na identificação dos sinais e sintomas, conduzindo o raciocínio clínico padronizado para a elaboração de DE apropriados, intervenção de enfermagem assertiva, com a possibilidade de mensurar os resultados da assistência, para favorecer a qualidade e segurança nos cuidados no neonato (AVENA MJ, et al., 2014).

\section{CONSIDERAÇÕES FINAIS}

O presente estudo permitiu revelar a partir dos artigos analisados as diferentes e oportunas contribuições das características definidoras para a proposição adequada do DE. Com base nas evidências científicas, sugere-se que a expertise do enfermeiro em examinar o RN, identificar sinais e sintomas e delinear as características definidoras direciona para a implementação de intervenções apropriadas e qualidade da assistência a estas crianças hospitalizadas. Nesta investigação acredita-se que uma limitação é a escassez de estudos voltados para a enfermagem na área neonatal, sobre características definidoras e os $\mathrm{DE}$, sendo que dos estudos encontrados a grande maioria foi desenvolvido com outras faixas etárias pediátricas.

\section{REFERÊNCIAS}

1. ALVARENGA SC, et al. Critical defining characteristics for nursing diagnosis about ineffective breastfeeding. Revista Brasileira de Enfermagem, 2018; 71 (2):314-321.

2. AQUINO WKM, et al. Accuracy of the defining characteristics in nursing diagnoses of Hyperthermia in newborns. Revista Brasileira de Enfermagem, 2018; 71(2):357-362.

3. AVENA MJ, et al. Conceptual validation of the defining characteristics of respiratory nursing diagnoses in neonates. Acta Paulista de Enfermagem, 2014; 27(1):76-85.

4. BRAGA FC, et al. Acurácia dos indicadores clínicos dos diagnósticos de enfermagem hipertermia e hipotermia em recémnascidos. Revista da Rede de Enfermagem do Nordeste, 2014;15(5):789-795.

5. CONSELHO FEDERAL DE ENFERMAGEM (COFEN).2009. Resolução COFEN n 358/2009. Dispõe sobre a Sistematização da Assistência de Enfermagem e a implementação do Processo de Enfermagem em ambientes, públicos ou privados, em que ocorre o cuidado profissional de Enfermagem, e dá outras providências. Brasília (DF): COFEN.

6. DANTAS AVVC, et al. Nursing Diagnosis of Neonatal Jaundice: study of clinical indicators. Journal of Pediatric Nursing, 2018; 39: e6-e10.

7. FREITAS LJQ, et al. Amamentação ineficaz entre nutrizes atendidas em unidades básicas de saúde. Revista enfermagem UERJ, 2014; 22(1): 103-110.

8. GALVÃO TF, et al. Principais itens para relatar Revisões sistemáticas e meta-análises: a recomendação PRISMA. Epidemiologia e Serviços de Saúde, 2015; 24(2): 335-342.

9. HERDMAN TH, KAMITSURU S. Diagnósticos de Enfermagem da NANDA-I: definições e classificação 2018-2020. 11. ed. Porto Alegre: Artmed, 2018; 459p.

10. MACHADO SPC, et al. Conhecimento, atitude e prática sobre fototerapia entre profissionais de enfermagem de hospitais de ensino. Revista Brasileira de Enfermagem, 2012; 65(1): 34-41.

11. MENDES KDS, et al. Revisão Integrativa: método de pesquisa para incorporação de evidências na saúde e na enfermagem. Texto \&Contexto - Enfermagem, 2008; 17(4): 758-764.

12. MONTEIRO FPM, et al. Validação clínica do diagnóstico de enfermagem "Disposição para desenvolvimento melhorado do lactente". Revista Brasileira de Enfermagem, 2016; 69(5): 855-863.

13. NASCIMENTO AMR, et al. Atuação do enfermeiro da estratégia saúde da família no incentivo ao aleitamento materno durante o período pré-natal. Revista Eletrônica Acervo Saúde,2019; (21): e667.

14. OLIVEIRA SIM, et al. Diagnósticos de enfermagem em recém-nascido com alterações glicêmicas. Revista Cogitare Enfermagem, 2013; 18(4): 702-708.

15. PARKER L, LUNNEY M. Moving beyond content validation of Nursing Diagnoses. Nursing Diagnosis, $1998 ; 9(4): 144-150$.

16. SANTOS CMC, et al. A estratégia PICO para construção da pergunta de pesquisa e busca de evidências. Revista LatinoAmericana de Enfermagem, 2007; 15(3):508-511.

17. SENA DTC, et al. A importância da atuação do enfermeiro no tratamento da icterícia neonatal. Revista Eletrônica Estácio Saúde, 2015; 4(2): 160-170.

18. SILVEIRA UA, et al. Características definidoras dos diagnósticos de enfermagem desobstrução ineficaz das vias aéreas e padrão respiratório ineficaz em crianças asmáticas. Revista da Rede de Enfermagem do Nordeste, 2008; 9(4): $125-133$.

19. TRUPPEL TC, et al. Sistematização da Assistência de Enfermagem em Unidade de Terapia Intensiva. Revista Brasileira de Enfermagem, 2009; 62(2): 221-227.

20. URAKAWA IT, KOBAYASHI RM. Identificação do perfil e diagnósticos de enfermagem do neonato com cardiopatia congênita. Revista de Pesquisa: cuidado é fundamental Online, 2012; 4(4): 3118-3124.

21. URSI ES. Prevenção de lesões de pele no perioperatório: revisão integrativa da literatura. Dissertação (Mestrado em Enfermagem) - Escola de Enfermagem de Ribeirão Preto. Universidade de São Paulo, Ribeirão Preto, $2005 ; 128$ p. 